\title{
Relações entre Parentalidade e Funções Executivas: Uma Revisão
}

\author{
Sistemática \\ Wayson Maturana de Souza* \\ Pontifícia Universidade Católica do Rio de Janeiro - PUC-Rio, Rio de Janeiro, RJ, Brasil \\ ORCID: https://orcid.org/0000-0003-3367-6448 \\ Luiz Fellipe Dias da Rocha** \\ Universidade do Estado do Rio de Janeiro - UERJ, Rio de Janeiro, RJ, Brasil \\ ORCID: https://orcid.org/0000-0001-9337-894X \\ Rafael Vera Cruz de Carvalho*** \\ Universidade do Estado do Rio de Janeiro - UERJ, Rio de Janeiro, RJ, Brasil \\ ORCID: https://orcid.org/0000-0002-4591-7993 \\ Ana Carolina Monnerat Fioravanti**** \\ Universidade Federal Fluminense - UFF, Niterói, RJ, Brasil \\ ORCID: https://orcid.org/0000-0003-2340-4043
}

\section{RESUMO}

A presente revisão sistemática investigou a produção científica a respeito da relação entre práticas parentais e funções executivas de crianças com desenvolvimento típico de zero a 13 anos. Foram realizadas buscas no Google Acadêmico, PsycNET, PubMed e SciELO, considerando artigos publicados entre 2008 e 2018, em português, inglês ou espanhol. Um total de 668 arquivos foi encontrado e, após as etapas de triagem, elegibilidade e seleção, foram incluídos 37 trabalhos. Os aspectos gerais dos artigos selecionados e seus resultados foram analisados. A maioria dos estudos foi realizada nos EUA e em países europeus, com crianças de zero a seis anos de idade. Para acessar as variáveis de interesse, diferentes métodos foram utilizados, como tarefas, testes, escalas e entrevistas. As práticas parentais investigadas foram categorizadas em: a) construtos gerais de parentalidade, b) parentalidade estimuladora, c) cuidados e afetividade parental e d) outros comportamentos parentais. Os resultados dos artigos indicaram relação entre as diferentes práticas parentais e as funções executivas dos filhos, sugerindo a influência da parentalidade no desenvolvimento executivo durante a infância. Orientações para estudos futuros são apresentadas.

Palavras-chave: relações pais-criança, relações familiares, desenvolvimento infantil, funções mentais, neuropsicologia. 


\title{
Relations between Parenting and Executive Functions: A Systematic
}

\section{Review}

\begin{abstract}
This systematic review investigated the scientific production regarding the relationship between parenting practices and executive functions of children with typical development from zero to 13 years old. Searches were made on Google Scholar, PsycNET, PubMed and SciELO, considering articles published between 2008 and 2018, in Portuguese, English or Spanish. A total of 668 files were found and, after the screening, eligibility and selection steps, 37 papers were included. The general aspects of the selected articles and their results were analyzed. Most studies were conducted in the USA and in European countries, with children from zero to six years old. To assess the variables of interest, different methods were used, such as tasks, tests, scales and interviews. The investigated parenting practices were categorized into: a) general parenting constructs, b) stimulating parenting, c) affective parental care, and d) other parenting behaviors. The results of the articles indicated a relationship between different parenting practices and the executive functions of children, suggesting the influence of parenting on executive development during childhood. Orientations for future studies are presented.
\end{abstract}

Keywords: parent child relations, family relations, child development, mental functions, neuropsychology.

\section{Relaciones entre las Prácticas Parentales y las Funciones Ejecutivas: Una}

\section{Revisión Sistemática}

\section{RESUMEN}

Esta revisión sistemática investigó la producción científica con respecto a la relación entre las prácticas parentales y las funciones ejecutivas de los niños con un desarrollo típico de cero a 13 años. Se realizaron búsquedas en Google Scholar, PsycNET, PubMed y SciELO, teniendo en cuenta los artículos publicados entre 2008 y 2018, en portugués, inglés o español. Se encontraron un total de 668 archivos y, después de los pasos de cribado, elegibilidad y selección, se incluyeron 37 documentos. Se analizaron los aspectos generales de los artículos seleccionados y sus resultados. La mayoría de los estudios se llevaron a cabo en los Estados Unidos y en países europeos, con niños de cero a seis años. Para acceder a las variables de interés, se utilizaron diferentes métodos, como tareas, pruebas, escalas y entrevistas. Las prácticas parentales investigadas se clasificaron en: a) constructos generales de parentalidad, b) parentalidad estimuladora, c) cuidados y afectividad parental y d) otros comportamientos parentales. Los resultados de los artículos indicaron una relación entre las diferentes prácticas parentales y las funciones ejecutivas de los niños, lo que sugiere la influencia de las prácticas parentales en el desarrollo ejecutivo durante la infancia. Se presentan orientaciones para futuros estudios.

Palabras clave: relaciones padres-niños, relaciones familiares, desarrollo infantil, funciones mentales, neuropsicología. 
As funções executivas (FEs) podem ser definidas como habilidades cognitivas de alta ordem, responsáveis pela capacidade de manter, gerenciar, planejar e otimizar informações, possibilitando aos indivíduos resolver problemas, tomar decisões e alcançar metas e objetivos específicos (Finegood \& Blair, 2017). Estas habilidades são essenciais para muitos aspectos do cotidiano, para a manutenção da saúde física, mental e para bons resultados acadêmicos (Diamond, 2016). Embora haja divergência na literatura em relação a quantas e quais são as FEs, um número considerável de autores concorda com a existência de três fatores: o controle inibitório, a memória operacional e a flexibilidade cognitiva (e.g., Diamond, 2016; Finegood \& Blair, 2017). O controle inibitório é o fator responsável pelo autocontrole, inibição de respostas prepotentes e supressão de distratores e de comportamentos e emoções desviantes do objetivo alvo (Diamond, 2016). A memória operacional é a responsável por manter e manipular informações por um período de tempo (Zelazo, 2015). Por fim, a flexibilidade cognitiva, cumpre a função de alternar estratégias, ações ou pensamentos entre dois ou mais cenários de processamento cognitivo distintos, flexibilizando respostas frente às diferentes demandas (Diamond, 2016; Zelazo, 2015).

Essas habilidades possuem relações com as áreas pré-frontais do cérebro, e seu desenvolvimento percorre a infância e adolescência, chegando ao ápice no início da fase adulta (Finegood \& Blair, 2017). Neste percurso, a interação entre a criança e o meio ambiente influencia o amadurecimento pré-frontal, possibilitando que as redes neuronais que sustentam o funcionamento executivo sejam consolidadas (Diamond, 2016; Finegood \& Blair, 2017). Dentre os fatores ambientais que favorecem o desenvolvimento das FEs, aponta-se: o status socioeconômico (e.g., exposição a violência, a toxinas e estimulações cognitivas no lar) fatores pré-natais (e.g., prematuridade, carência de determinados nutrientes durante a gravidez) e as interações parentais entre os pais e as crianças (Carlson, 2009; Hackman, Farah, \& Meaney, 2010).

Hoghughi (2004) define o conceito de parentalidade como um conjunto de atividades intencionais necessárias à sobrevivência e que fornecem cuidado, desenvolvimento e bemestar às crianças. Segundo os autores, a parentalidade envolve diferentes funções relacionadas, que podem mudar de forma gradual ao longo do desenvolvimento da criança. Assim, o reconhecimento de uma ampla diversidade de práticas parentais (e.g., atividades, controle e cuidados parentais) é característica marcante no estudo do tema (Hoghugui, 2004). Macarini, Martins, de Minetto e Vieira (2010), em uma revisão da literatura brasileira, apontaram que os estudos sobre práticas parentais podem ser agrupados em três categorias: as práticas de cuidado, mais pesquisadas durante a primeira (dos zero aos três anos) e segunda 
infância (dos quatro aos seis anos); as práticas educativas, pesquisadas desde a primeira infância e com destaque na adolescência (entre 12 e 20 anos); e os estilos parentais, mais pesquisados em relação a adolescência e a fase adulta.

As práticas parentais exercem papel crucial no amadurecimento cerebral e cognitivo das crianças, seja através da estimulação direta ou exercendo forças moduladoras sobre o ambiente experimentado por elas (Britto et al., 2017; Hackman et al., 2010). As práticas parentais são apontadas por alguns estudos como importantes influências sociais para o desenvolvimento das FEs das crianças (e.g., Carlson, 2009; Finegood \& Blair, 2017; Landry, Miller-Loncar, Smith, \& Swank, 2002). Assim também, as práticas de suporte durante a resolução de problemas em brincadeiras e jogos, podem ser influenciadoras do desenvolvimento executivo (Landry et al., 2002). Além disso, as práticas parentais também tem sido relacionadas a um menor nível de cortisol basal da criança e a um melhor desenvolvimento da autorregulação e do controle inibitório (Finegood \& Blair, 2017). Em contrapartida, dificuldades provocadas pelo baixo status socioeconômico (e.g., ausência de recursos, tempo disponível para a criança e estresse parental) tendem a diminuir a qualidade das práticas parentais e esta carência parece impactar no desenvolvimento das FEs (Finegood \& Blair, 2017; Hackman et al., 2010).

Nesse sentido, o presente trabalho apresenta uma revisão sistemática de artigos (publicados entre 2008 e 2018) sobre as relações entre práticas parentais e o desenvolvimento infantil das FEs. Há uma revisão sistemática em língua portuguesa destinada a este tema (Doellinger, Soares, Sampaio, Mesquita, \& Baptista, 2017), mas, no entanto, é dedicada a avaliar estudos sobre parentalidade com crianças a termo e prematuras. Em contraponto, o presente estudo visou investigar as relações entre as práticas parentais e o desenvolvimento das FEs em crianças de desenvolvimento típico.

\section{Método}

Optou-se por realizar uma revisão sistemática, pois esta permite uma busca mais refinada de artigos, localizando o maior número possível de resultados, diminuindo vieses subjetivos na escolha dos textos e deixando explícito aos leitores critérios e etapas utilizados (Costa \& Zoltowski, 2014).

Para acessar os artigos foram utilizados os seguintes bancos: Google Acadêmico, PsycINFO, PubMed e SciELO. As buscas foram feitas utilizando palavras-chave relevantes ao tema nos idiomas inglês, português e espanhol (ver Figura 1). A escolha das palavras- 
chave, assim como de suas traduções, se deu com auxílio dos bancos de terminologia científicas da APA (American Psychological Association - Thesaurus of Psychological Index Itens), da Biblioteca Virtual em Saúde (BVS, Descritores em Ciências da Saúde - DeCS) e da U. S. National Library of Medicine (NLM, Medical Subject Headings - MeSH). Para resgatar artigos relevantes e recentes, a pesquisa foi limitada a palavras-chave pesquisadas apenas pelo título das publicações, compreendendo o período entre 2008 e 2018.

Durante a busca, foram localizados 668 arquivos e estes foram avaliados a fim de eliminar duplicatas e trabalhos que não fossem artigos revisados por pares (e.g., teses, dissertações, capítulos de livros). Em seguida, foram aplicados os critérios de inclusão e exclusão. Para a inclusão, foi estabelecido: estudos que contemplaram a relação entre parentalidade e FEs em crianças de desenvolvimento típico, quando os construtos de parentalidade foram avaliados até 13 anos de idade. Artigos avaliando as FEs em idade superior a 13 anos, também foram incluídos. Como critério de exclusão, foram eliminados artigos que abordaram: a) práticas parentais de alimentação; b) influências dos pais que não correspondiam a cuidados e interações (e.g., influências genéticas, influências pré-natais, escolaridade parental); c) crianças prematuras ou de baixo peso ao nascimento; d) pais com transtornos psicológicos; e) eficácia de intervenções; f) amostras com (apenas) crianças vítimas de abuso, punições físicas ou maus tratos; g) revisões e artigos teóricos.

Ao fim das análises, 39 artigos foram selecionados para leitura. Dois artigos foram removidos devido à impossibilidade de acesso, restando 37 artigos aptos a integrar a revisão. As etapas de busca e seleção dos artigos foram realizadas por dois juízes de maneira independente. Nos casos de discordâncias sobre a inclusão de artigos, um terceiro juiz-cego foi responsável por decidir a permanência ou não do estudo. 


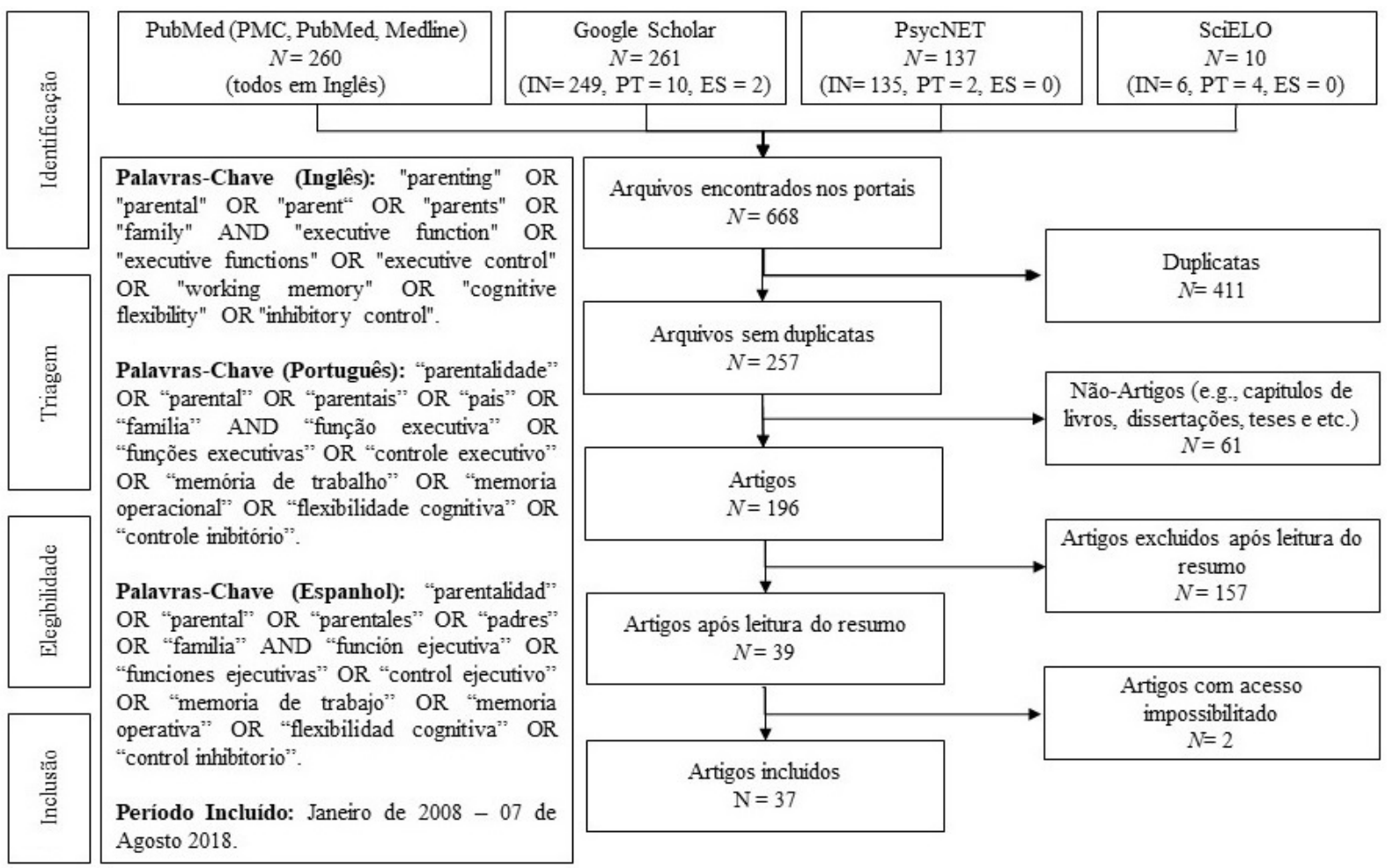

Figura 1. Diagrama de fluxos de pesquisa e seleção de artigos. Nota. IN = inglês; PT = português; ES = espanhol.

\section{Resultados}

Os 37 artigos selecionados foram avaliados com base nas seguintes categorias: aspectos gerais dos estudos (contexto geográfico, delineamento dos estudos, características dos participantes e instrumentos utilizados) e resultados dos estudos (componentes das FEs, tipos de parentalidade e relações encontradas entre os construtos).

\section{Aspectos gerais dos estudos}

Os Estados Unidos realizaram o maior número de publicações da amostra com 51,3\% ( $\mathrm{n}=19)$, seguidos pela Holanda com 10,8\% ( $\mathrm{n}=4)$ e Inglaterra e Canadá com 8,1\% $(\mathrm{n}=3)$ cada. A China produziu 5,4\% $(\mathrm{n}=2)$ dos artigos da amostra e a Austrália, a Bélgica, o Brasil, o Chile, a Coréia do Sul e a Polônia apenas $2,7 \%(\mathrm{n}=1)$ cada.

Todos os trabalhos incluídos apresentaram pesquisas quantitativas. Do total de artigos, 59,5\% ( $\mathrm{n}=22)$ foram estudos de desenho longitudinal e 40,5\% ( $\mathrm{n}=15)$ de desenho transversal. Dos 15 estudos transversais, que estudaram as relações entre práticas parentais e FEs no mesmo recorte temporal, $26,67 \%(n=4)$ acessaram os construtos em crianças entre 
zero e três anos, 33,33\% ( $\mathrm{n}=5)$ em crianças entre três e seis anos, $20 \%(\mathrm{n}=3)$ em crianças acima de seis anos, 6,67\% $(\mathrm{n}=1)$ em crianças entre dois e cinco anos e 6,67\% (n=1) em crianças entre quatro e oito anos. Um estudo $(6,67 \%)$ não especificou a faixa etária acessada (informando que as crianças estavam no "segundo ano básico") (Bernal-Ruiz, RodríguezVera, González-Campos, \& Torres-Álvarez, 2017). Nos 22 estudos longitudinais, as práticas parentais e as FEs foram acessadas em momentos distintos sendo, 45,45\% ( $\mathrm{n}=10)$ das práticas parentais acessadas em crianças dos zero aos três anos, 45,45\% ( $\mathrm{n}=10)$ em crianças dos três aos seis anos, e 9,09\% $(n=2)$ em uma faixa que compreendeu desde os zero até os seis anos de idade. Em relação aos mesmos estudos longitudinais, as FEs foram acessadas em 13,64\% $(n=3)$ dos trabalhos em crianças entre zero e três anos, em $81,82 \%(n=18)$ em crianças entre três e seis anos e em um estudo (4,55\%) durante a adolescência.

Para acessar os diferentes tipos de comportamentos parentais, 25 estudos utilizaram observações de interações diádicas frente a tarefas como quebra-cabeças e/ou atividades de resolução de problemas e 12 estudos utilizaram escalas preenchidas pelos pais e cuidadores e um usou escala preenchida pela própria criança. Cinco estudos usaram entrevistas com os pais para investigar variáveis parentais. Seis estudos utilizaram mais de uma categoria de instrumentos para avaliar os comportamentos parentais. Os construtos de FEs também foram avaliados de maneira relativamente heterogênea pelos artigos, sendo utilizado uma gama de instrumentos para acessá-los. Embora os instrumentos escolhidos em cada estudo para avaliar as habilidades executivas tenham variado de maneira expressiva, 81,08\% ( $\mathrm{n}=30)$ dos trabalhos utilizaram tarefas e testes, enquanto $16,22 \%(n=6)$ utilizaram escalas. Apenas um estudo $(2,70 \%)$ usou testes e escala, para acessar comportamentos relacionados as FEs na segunda infância (Meuwissen \& Englund, 2016).

\section{Resultados dos estudos}

Fatores únicos de FEs foram avaliados em 18 estudos (embora muitos fossem compostos por instrumentos de fatores específicos). Os fatores nucleares (i.e., memória operacional, controle inibitório e/ou flexibilidade cognitiva) foram avaliados por 19 artigos. A metacognição emergente (composta por itens de planejamento, memória operacional e flexibilidade cognitiva da escala "Behavior Rating Inventory of Executive Function" BRIEF) foi avaliada em três artigos, o controle emocional (fator específico da escala BRIEF) em um artigo, o planejamento (fator específico da escala BRIEF) em dois artigos, FEs de conflito (composto de tarefas de memória operacional e controle inibitório atencional) e FEs 
de controle do impulso (relacionada a capacidade de controlar respostas impulsivas frente a cenários de recompensas) em um estudo. Dois trabalhos usaram os instrumentos separadamente como medidas de FEs (i.e., Kamza, Putko, \& Złotogórska, 2016; Martins, León, \& Seabra, 2016), sendo que o primeiro visou avaliar as FEs frias (funções puramente cognitivas) e FEs quentes (relacionadas a aspectos afetivos e motivacionais).

Um resultado que chama atenção é a heterogeneidade dos construtos sobre parentalidade avaliados pelos estudos. Visando apresentar os conceitos de parentalidade de maneira didática, foi realizada uma classificação dos artigos a partir de quatro categorias de práticas parentais: a) construtos gerais de parentalidade, b) parentalidade estimuladora, c) cuidados e afetividade parental e d) outros comportamentos parentais. Antes de apresentar cada classificação, é preciso esclarecer que as categorias delineadas são arbitrárias e baseadas em pontos comuns nas definições dos construtos, havendo discordâncias nas delimitações dos conceitos por alguns autores. Um total de 45,95\% $(n=17)$ dos estudos avaliou mais de um comportamento parental, podendo assim o mesmo estudo constar em mais de uma categoria.

A categoria denominada de construtos gerais de parentalidade corresponde a estudos que utilizaram fatores latentes gerais (não diretamente observáveis) para simplificar um conjunto complexo de dados derivados de comportamentos parentais específicos. Como exemplo desta redução de dados, Sulik, Blair, Mills-Koonce, Berry e Greenberg, (2015) transformaram avaliações de práticas parentais, como: sensibilidade, desapego (score reverso), estimulação, consideração positiva (positive regard) e animação; avaliados através de observação de interação diádica dos seis aos 36 meses, em um fator único, chamado de qualidade parental (o mesmo foi realizado aos cinco anos, transformando múltiplos fatores em uma medida única de qualidade parental). Rolan, Schmitt, Purpura e Nichols (2018) abordaram um construto de parentalidade global, e de modo semelhante, Meuwissen e Englund (2016), Amicarelli, Kotelnikova, Smith, Kryski e Hayden (2018) e Blair, Cybele Raver e Berry (2014), reduziram dados de múltiplas práticas parentais a um fator único, denominando-os de qualidade parental (para os primeiros autores) e parentalidade positiva (para os outros dois estudos), respectivamente. Alguns artigos utilizaram a redução de dados a dois fatores latentes: um abordando diversas práticas parentais positivas (e.g. sensibilidade, suporte e presença); e outro condensando práticas parentais negativas (e.g. intrusividade e severidade parental) (Blair et al., 2011; Smith, Kryski, Sheikh, Singh, \& Hayden, 2013). Embora não tenham apresentado uma medida composta por redução de múltiplas variáveis, Devine, Bignardi e Hughes (2016) e Hughes e Devine (2017), em trabalhos utilizando a mesma amostra, apresentaram uma medida generalista de interações negativas entre pais e 
crianças (negative parent-child interactions) que contemplava aspectos como controle negativo, afetos negativos e conflitos com a criança.

A segunda categoria, denominada de parentalidade estimuladora, concentra comportamentos parentais que visavam auxiliar a performance de crianças em tarefas, promovendo seu desenvolvimento cognitivo e a autonomia. Podemos dividir estas categorias em dois tipos: o apoio de autonomia (autonomy support) e outros comportamentos de estimulação cognitiva. Há diferentes termos com definições semelhantes ao apoio de autonomia (e.g., scaffolding, guidance e encouragement of autonomy) e todas estas definições foram consideradas. Nessa categoria foram incluídos estudos que abordaram o apoio verbal dado pelos pais durante a execução de uma tarefa, facilitando o êxito da criança (Bibok, Carpendale, \& Müller, 2009; Bindman, Hindman, Bowles, \& Morrison, 2013; Spruijt, Dekker, Ziermans, \& Swaab, 2018); assim como trabalhos abordando o apoio verbal e não verbal observado nas interações entre cuidadores e crianças (Bindman, Pomerantz, \& Roisman, 2015; Cheng, Lu, Archer, \& Wang, 2018; Cipriano-Essel, Skowron, Stifter, \& Teti, 2013; Devine et al., 2016; Eason \& Ramani, 2017; Hammond, Müller, Carpendale, Bibok, \& Liebermann-Finestone, 2012; Hughes \& Devine, 2017; Lee, Baker, \& Whitebread, 2018; Meuwiseen \& Carlson, 2015). Em relação aos comportamentos de estimulação cognitiva, que podemos definir como os esforços para ensinar ou facilitar o desenvolvimento das crianças, pode-se classificar as medidas de ambiente de aprendizado domiciliar (home learning environment - HLE) apresentadas nos estudos de Devine et al. (2016) e Hughes e Devine (2017). Os comportamentos de realização de atividades de enriquecimento, de incentivo a maturidade e de oportunidade de aprendizado, apresentados por Sarsour et al. (2011), também foram adicionados a esta categoria.

A terceira categoria, descrita como práticas de cuidado e afetividade parental, foca em comportamentos que envolvem questões emocionais e de prontidão para o cuidado exercidas em relação à criança. Como parte desta categoria, pode-se observar comportamentos de parentalidade com conotação positiva, como: sensibilidade (sensitivity) (Blair et al., 2014; Cheng et al., 2018; Gueron-Sela et al., 2017; Holochwost et al., 2016; Holochwost, Volpe, Gueron-Sela, Propper, \& Mills-Koonce 2018; Kok et al., 2014; Lucassen et al., 2015; MilevaSeitz et al., 2015; Spruijt et al., 2018), calorosidade (warmth) (Berthelsen, Hayes, White, \& Williams, 2017; Lam, Chung, \& Li, 2018; Vandenbroucke, Spilt, Verschueren, \& Baeyens, 2017), responsividade (responsiveness) (Blair et al., 2014; Merz, Landry, Montroy, \& Williams, 2017; Sarsour et al., 2011; Vernon-Feagans, Willoughby, Garrett-Peters, \& Family Life Project Key Investigators, 2016), assim como comportamentos de parentalidade com 
conotação negativa: severidade (harsh) (Lucassen et al., 2015; Treat, Sheffield Morris, Williamson, Hays-Grudo, \& Laurin, 2017), conflito (Vandenbroucke et al., 2017), raiva (anger) (Berthelsen et al., 2017), intrusividade negativa (negative intrusiveness) (Holochwost et al., 2016; Holochwost et al., 2018) e hostilidade parental (hostility) (Lam et al., 2018). A classificação em conotação positiva e negativa se deu em função dos comportamentos parentais promoverem cuidados e estarem ligados a afetos de valência positiva (conotação positiva) ou serem punitivos, desestimuladores de comportamentos das crianças e relacionados a afetos de valência negativa (conotação negativa).

Ainda nesta categoria, Cheng et al. (2018) abordaram um construto parental positivo denominado de mind-mindedness, o qual os autores definem como a tendência parental de descrever de maneira reflexiva as características inferidas através da sensibilidade e da resposta frente às demandas do bebê. Deste modo, diferentemente da sensibilidade, no comportamento de mind-mindedness, os pais respondem à demanda da criança (e.g., fome) e dão um feedback verbal acerca de um estado mental inferido, projetando através da fala uma compreensão da criança acerca daquele fenômeno. Outros comportamentos de cuidado como a disciplina (Kok et al., 2014), consistência do cuidado parental (Berthelsen et al., 2017), a indução de culpa (Yu, Cheah, Hart, \& Yang, 2018), o companheirismo e a integração familiar (Sarsour et al., 2011) também foram associados a este seguimento.

Por fim, a categoria denominada de outros comportamentos parentais contempla construtos de parentalidade que não puderam ser classificados nas demais categorias. Fazem parte deste seguimento construtos menos frequentes nesta revisão, como os trabalhos envolvendo competências parentais (Bernal-Ruiz et al., 2017), estilos parentais (Martins, León, \& Seabra, 2016), atitudes parentais (Kamza et al., 2016) e a parentalidade ansiogênica, ou seja, que aumenta a ansiedade da criança (e.g., modelagem, envolvimento excessivo e acomodação dos pais) (Brennan, Luke, Murphy, Francazio, \& Flessner, 2018).

Perante a relação entre práticas parentais e FEs, a maior parte dos estudos apontou alguma influência das práticas parentais no desenvolvimento executivo. Apenas dois estudos, um sobre parentalidade ansiogênica (Brennan, Luke, Murphy, Francazio, \& Flessner, 2018) e outro sobre indução de culpa (Yu et al., 2018), não apontaram nenhuma relação entre os conceitos. Embora as interações entre práticas parentais e FEs tenham sido evidentes, nem todos os construtos de parentalidade apresentaram esta relação. Relações entre as FEs e a categoria "construtos gerais de parentalidade" e o apoio de autonomia, da categoria "parentalidade estimuladora" foram as mais evidentes. Já a categoria "práticas de cuidado e afetividade parental" obteve resultados menos claros. Na categoria "outros comportamentos 
parentais", apenas o construto "parentalidade ansiogênica" (Brennan et al., 2018) não apontou nenhuma relação com as FEs. Para além do fator geral das FEs, o controle inibitório foi o fator nuclear mais pesquisado e significativamente associado às práticas parentais.

Em relação à categoria "construtos gerais de parentalidade", os fatores latentes de qualidade parental (Meuwissen \& Englund, 2016; Rolan et al., 2018; Sulik et al., 2015) e parentalidade positiva (Amicarelli et al., 2018; Blair et al., 2011; Smith et al., 2013) estiveram associados com uma melhor habilidade executiva, enquanto os componentes de parentalidade negativa apresentaram relações inversas ao desempenho das FEs em todos os estudos (Blair et al., 2011; Devine et al., 2016; Hughes \& Devine, 2017; Smith et al., 2013).

$\mathrm{Na}$ categoria "parentalidade estimuladora", os estudos abordando o apoio de autonomia e construtos semelhantes, se mostraram positivamente relacionados as FEs na maior parte dos trabalhos. Apenas o estudo de Kamza et al.(2016) apontou um resultado conflitante, visto que um maior apoio de autonomia se correlacionou com um menor escore de flexibilidade cognitiva em crianças com idades entre três e quatro anos. Cabe destacar que o estudo de Kamza et al. (2016) avaliou a atitude de apoio de autonomia materna, utilizando uma escala de autorrelato, o que contrasta com os demais estudos, que acessaram o fenômeno através da observação de interações diádicas. Ao que tange os comportamentos parentais de estimulação, Devine et al. (2016) e Hughes e Devine (2017) não evidenciaram efeito do ambiente de aprendizado domiciliar nas FEs de crianças de cinco anos. As práticas de atividades de enriquecimento, e de oportunidade de aprendizado (mas não o incentivo a maturidade) se mostraram positivamente relacionados às FEs (Sarsour et al., 2011).

Quanto à categoria "práticas de cuidado e afetividade parental", a sensibilidade se mostrou associada positivamente às FEs em parte dos estudos (Blair et al., 2014; Gueron-Sela et al., 2017; Holochwost et al., 2018; Kok et al., 2014; Lucassen et al., 2015; Spruijt et al., 2018); porém, Cheng et al. (2018), em uma amostra de crianças chinesas de seis a 38 meses, não apontaram relação entre as variáveis. De modo semelhante, Holochwost et al. (2016) evidenciaram relações entre a sensibilidade e as FEs apenas em crianças euro-americanas, não obtendo o mesmo efeito em crianças afro-americanas, o que parece apontar para um efeito transcultural. Efeitos de gênero foram achados no estudo longitudinal de Mileva-Seitz et al. (2015) com crianças acompanhadas dos 14 aos 52 meses, apontando maior suscetibilidade dos meninos à sensibilidade parental, sendo esta correlacionada com melhor controle inibitório neste grupo. Em relação à calorosidade, Berthelsen et al. (2017) encontraram influência indireta nas FEs de adolescentes australianos entre 14 e 15 anos, enquanto Lam et al. (2018) não observaram tais associações em crianças chinesas de quatro e seis anos. 
Vandenbroucke et al. (2017) identificaram efeito inversamente proporcional da baixa calorosidade na memória operacional de crianças entre seis e oito anos (efeitos positivos de alta calorosidade não foram apontados).

Para a variável hostilidade, Lam et al. (2018) observaram relações negativas com as FEs, tanto para hostilidade materna, quanto paterna. Também foram identificadas relações positivas entre responsividade e FEs em todos os estudos abordando esta variável (Blair et al., 2014; Merz et al., 2017; Sarsour et al., 2011; Vernon-Feagans et al., 2016). Outros comportamentos pertencentes à categoria de cuidado e afetividade parental, como disciplina (Kok et al., 2014), consistência do cuidado (de forma indireta) (Berthelsen et al., 2017), companheirismo familiar (Sarsour et al., 2011) e mind-mindedness (Cheng et al., 2018), também apresentaram relações positivas com as FEs. Os comportamentos com conotação negativa de severidade parental (atos coercivos, emocionalmente negativos e disciplina negativa), conflito (oposição parental ao comportamento da criança) e raiva (sentimentos de raiva e frustração na interação), foram associados a maiores problemas executivos das crianças (Berthelsen et al., 2017; Lucassen et al., 2015; Treat, Sheffield Morris, Williamson, Hays-Grudo, \& Laurin, 2017; Vandenbroucke et al., 2017).

Para a categoria "outros comportamentos parentais", três dos quatro estudos mostraram relações entre parentalidade e FEs. Em um estudo referente à atitude materna, Kamza et al. (2016) identificaram relações positivas entre a atitude protetiva e o controle inibitório, e relações negativas entre a atitude de apoio de autonomia e a flexibilidade cognitiva de crianças entre três e quatro anos. O trabalho de Bernal-Ruiz et al. (2017), encontrou relações positivas entre as competências parentais protetoras, reflexivas e formativas no desenvolvimento das FEs. E, por fim, o artigo de Martins et al. (2016) apontou correlações de magnitude baixa a moderada entre o fator inconsistência de punição (de uma escala de estilos parentais) e um maior tempo de reação nas etapas incongruentes do teste Stroop (medida de controle inibitório atencional); assim como relações inversamente proporcionais com uma maior flexibilidade cognitiva (medida pela parte B do teste de trilhas). O estudo sobre parentalidade ansiogênica em crianças e adolescentes de nove a 17 anos (Brennan et al., 2018) não observou nenhuma interação com o fator geral das FEs. 


\section{Discussão}

Em relação aos 37 trabalhos analisados, 35 mostraram algum tipo de relação entre as práticas parentais e as FEs, sendo os trabalhos de Brennan et al. (2018) sobre a parentalidade ansiogênica e o de Yu et al. (2018) sobre a indução de culpa os únicos estudos a não apresentarem nenhuma relação entre práticas parentais e FEs. Estes resultados concordam com os argumentos de Carlson (2009) e Finegood e Blair (2017) a respeito da influência social no desenvolvimento executivo, que por vezes é negligenciada nos trabalhos sobre a temática. Embora as interações entre práticas parentais e FEs tenham sido evidentes nos estudos, nem todas as práticas estudadas apresentaram estas relações. Tais resultados ocorrem porque uma variedade de práticas parentais distintas foi mensurada em diferentes faixas etárias e em contextos culturais heterogêneos. Ainda que os estudos tenham começado a identificar a primeira e segunda infância como um período relevante em que as práticas parentais parecem ter influência sobre as FEs, torna-se necessário identificar a janela temporal em que cada prática parental exerce maior impacto neste desenvolvimento.

Os estudos que se enquadraram na categoria "construtos gerais de parentalidade" evidenciaram que todos os construtos únicos de parentalidade, formados a partir do agrupamento de práticas parentais distintas (e.g., qualidade parental, parentalidade positiva e negativa), estiveram correlacionados com as FEs das crianças (e.g., Amicarelli et al., 2018; Blair et al., 2014, 2011; Sulik et al., 2015). A análise destes fatores únicos pode trazer a vantagem de identificar efeitos cumulativos e de interação de um grupo de práticas parentais no desenvolvimento executivo. Por outro lado, conforme apontado por Hoghughi (2004), a parentalidade consiste em um conjunto bastante heterogêneo de práticas, de execução e finalidade distintas e, sendo assim, os componentes únicos de parentalidade podem gerar imprecisão entre o protagonismo de cada comportamento parental no desenvolvimento das FEs. Contudo, a heterogeneidade da literatura a respeito pode ser positiva para entender tanto os efeitos particulares, como os efeitos de interação entre variáveis distintas.

Como visto, artigos sobre o apoio de autonomia e construtos semelhantes, classificados na categoria "parentalidade estimuladora", apontaram relações significativas com as FEs. Estes resultados corroboram os encontrados por Landry et al. (2002) em relação à influência do andaime parental aos três anos de idade nas habilidades executivas (planejamento e memória operacional) aos seis anos. Pode-se apontar como uma limitação dos artigos desse segmento a falta de consenso em relação à nomenclatura das variáveis (e.g., Bindman et al., 2015; Cheng et al., 2018; Eason \& Ramani, 2017; Hammond et al., 2012). 
Conforme apresentado, alguns autores abordam definições semelhantes para os construtos de apoio ou encorajamento de autonomia (autonomy support/ encouragement of autonomy) e andaime parental (parental scaffolding).

Na categoria "práticas de cuidado e afetividade parental", as relações entre as práticas parentais e as FEs foram um pouco mais dissonantes. Em relação à sensibilidade, enquanto alguns estudos (Blair et al., 2014; Holochwost et al., 2018; Kok et al., 2014; Lucassen et al., 2015) encontraram relações positivas com as FEs, outros estudos apontaram diferenças transculturais (Cheng et al., 2018; Holochwost et al., 2016) e de gênero (Mileva-Seitz et al., 2015). De modo semelhante, em relação à calorosidade (Berthelsen et al., 2017; Lam et al., 2018; Vandenbroucke et al., 2017), os três artigos diferem em idade de acesso das FEs e aos países de origem das pesquisas (Austrália, China e Bélgica, respectivamente), o que pode ter influenciado seus diferentes resultados.

Os comportamentos descritos como sensibilidade, responsividade e calorosidade apresentam similaridade em suas descrições. A sensibilidade é abordada em alguns estudos, como oposta à intrusividade, definida como o comportamento parental de colocar os próprios interesses e demandas em detrimento dos da criança, não reconhecendo seus desejos e intenções (Holochwost et al., 2016, 2018; Spruijt et al., 2018). A calorosidade parental está ligada às manifestações de afeto com conotação positiva dos pais em relação à criança, tendo por contraponto a hostilidade (Bindman et al., 2015; Lam et al., 2018). Já a responsividade parental diz respeito à consistência do envolvimento parental e o ato de dar respostas apropriadas às demandas da criança (Merz et al., 2017). Alguns trabalhos sobre responsividade englobam uma definição ampla, que pode abranger os conceitos de sensitividade, calorosidade e apoio de autonomia (Merz et al., 2017; Vernon-Feagans et al., 2016), evidenciando novamente a dificuldade de consenso entre os estudos referentes a estas práticas parentais.

Na categoria "outros comportamentos parentais", ressalta-se a presença dos únicos dois estudos sul-americanos da amostra (i.e., Bernal-Ruiz et al., 2017; Martins et al., 2016), o que aponta a necessidade de desenvolvimento de novos trabalhos investigando as relações entre parentalidade e FEs neste território. Ao comparar os resultados aqui obtidos com os da revisão de Macarini et al. (2010) sobre os estudos de práticas parentais no Brasil, pode-se notar que o trabalho de Martins et al.(2016) está em consonância com a investigação nacional a respeito das práticas parentais. Porém, em relação à literatura internacional, esta revisão aponta que o estudo das práticas educativas e estilos parentais parece incomum nos estudos 
relacionando práticas parentais e desenvolvimento das FEs (pelo menos em relação à idade pesquisada).

Embora muitos estudos tenham focado nos fatores generalistas das FEs, os resultados desta revisão sistemática foram condizentes com a argumentação de Diamond, (2016), Finegood e Blair (2017) e Zelazo (2015) a respeito da existência de três FEs nucleares, sendo estas analisadas em boa parte dos trabalhos. O destaque dado por parte dos trabalhos ao estudo do controle inibitório não surpreende, visto a relativa sobreposição entre o controle inibitório e a capacidade de autorregulação (Diamond, 2016), que por sua vez tem seu desenvolvimento associado às relações entre os pais e criança durante os primeiros anos de vida (Carlson, 2009; Finegood \& Blair, 2017).

É importante registrar como limitação que este estudo apresenta apenas os resultados dos artigos de modo qualitativo e, sendo assim, não é possível fazer inferências a respeito do tamanho do efeito da parentalidade nas FEs ou o quão robusto são os resultados obtidos a favor ou contra a hipótese. Apresentada tal limitação, é importante frisar que um ponto positivo desta revisão foi a preocupação em incluir artigos em português e espanhol, o que favoreceu a visibilidade da produção latino-americana, mesmo que tenham sido localizados apenas um estudo em cada uma destas línguas. Outro ponto a ser considerado é a carência de literatura nacional acerca do assunto, o que faz desta revisão um trabalho relevante para a difusão da temática em nosso contexto.

\section{Considerações Finais}

O objetivo deste trabalho foi realizar uma revisão sistemática dos estudos da última década (2008-2018) a respeito da influência das práticas parentais no desenvolvimento das FEs. Com base nisto, apesar da grande heterogeneidade dos estudos, é possível afirmar que os artigos contemplados nesta revisão evidenciam a existência de relação entre as variáveis, sugerindo a influência das práticas parentais no desenvolvimento executivo durante a infância. Entretanto, conforme apontam alguns trabalhos, é possível que haja relação bidirecional entre os construtos (Merz et al., 2017). Para resolver questões desta natureza, assim como clarificar efeitos transculturais e de gênero, futuros trabalhos são necessários. 


\section{Referências}

Amicarelli, A. R., Kotelnikova, Y., Smith, H. J., Kryski, K. R., \& Hayden, E. P. (2018). Parenting differentially influences the development of boys' and girls' inhibitory control. British Journal of Developmental Psychology, 36(3), 371-383. doi: 10.1111/bjdp. 12220

Bernal-Ruiz, F., Rodríguez-Vera, M., González-Campos, J., \& Torres-Álvarez, A. (2017). Competencias parentales que favorecen el desarrollo de funciones ejecutivas en escolares. Revista Latinoamericana de Ciencias Sociales, Niñez y Juventud, 16(1), 163-176. doi: 10.11600/1692715x.16109

Berthelsen, D., Hayes, N., White, S. L. J., \& Williams, K. E. (2017). Executive function in adolescence: Associations with child and family risk factors and self-regulation in early childhood. Frontiers in Psychology, 8, 1-14. doi: 10.3389/fpsyg.2017.00903

Bibok, M. B., Carpendale, J. I. M., \& Müller, U. (2009). Parental scaffolding and the development of executive function. New Directions for Child and Adolescent Development, 2009(123), 17-34. doi: 10.1002/cd.233

Bindman, S. W., Hindman, A. H., Bowles, R. P., \& Morrison, F. J. (2013). The contributions of parental management language to executive function in preschool children. Early Childhood Research Quarterly, 28(3), 529-539. doi: 10.1016/j.ecresq.2013.03.003

Bindman, S. W., Pomerantz, E. M., \& Roisman, G. I. (2015). Do Children's Executive Functions Account for Associations Between Early Autonomy-Supportive Parenting and Achievement Through High School? Journal of Educational Psychology, 107(3), 756-770. doi: 10.1037/edu0000017

Blair, C., Cybele Raver, C., \& Berry, D. J. (2014). Two approaches to estimating the effect of parenting on the development of executive function in early childhood. Developmental Psychology, 50(2), 554-565. doi: 10.1037/a0033647

Blair, C., Granger, D. A., Willoughby, M., Mills-Koonce, R., Cox, M., Greenberg, M. T., ... Fortunato, C. K. (2011). Salivary Cortisol Mediates Effects of Poverty and Parenting on Executive Functions in Early Childhood. Child Development, 82(6), 1970-1984. doi: 10.1111/j.1467-8624.2011.01643.x

Brennan, E., Luke, A., Murphy, Y., Francazio, S., \& Flessner, C. (2018). Examining the Relationship Between Anxiogenic Parenting Practices and Cognitive Flexibility in Youth. Behavior Modification, 2(6), 864-884. doi: 10.1177/0145445517748558 
Britto, P. R., Lye, S. J., Proulx, K., Yousafzai, A. K., Matthews, S. G., Vaivada, T., ... Bhutta, Z. A. (2017). Nurturing care: Promoting early childhood development. The Lancet, 389(10064), 91-102. doi: 10.1016/S0140-6736(16)31390-3

Carlson, S. M. (2009). Social origins of executive function development. New Directions for Child and Adolescent Development, 2009(123), 87-98. doi: 10.1002/cd.237

Cheng, N., Lu, S., Archer, M., \& Wang, Z. (2018). Quality of maternal parenting of 9-monthold infants predicts executive function performance at 2 and 3 years of age. Frontiers in Psychology, 8, 1-12. doi: 10.3389/fpsyg.2017.02293

Cipriano-Essel, E., Skowron, E. A., Stifter, C. A., \& Teti, D. M. (2013). Heterogeneity in Maltreated and Non-maltreated Preschool Children's Inhibitory Control: The Interplay Between Parenting Quality and Child Temperament. Infant and Child Development, 22(5), 501-522. doi: 10.1002/icd.1801

Costa, A. B. \& Zoltowski, A. P. C. (2014). Como escrever um artigo de revisão sistemática. In S. H. Koller, M. C. P. de Paula Couto, \& J. V. Hohendorff (Eds.), Manual de Produção Científica (pp. 55-70). Porto Alegre: Penso.

Devine, R. T., Bignardi, G., \& Hughes, C. (2016). Executive function mediates the relations between parental behaviors and children's early academic ability. Frontiers in Psychology, 7, 1-15. doi: 10.3389/fpsyg.2016.01902

Diamond, A. (2016). Why improving and assessing executive functions early in life is critical. In J. A. Griffin, P. McCardle, \& L. Freund (Eds.), Executive function in preschool-age children: Integrating measurement, neurodevelopment, and translational research (pp. 11-43), Washington: American Psychological Association. doi: 10.1037/14797002

Doellinger, P. V., Soares, I., Sampaio, A., Mesquita, A. R., \& Baptista, J. (2017). Prematuridade, Funções Executivas e Qualidade dos Cuidados Parentais: Revisão Sistemática de Literatura. Psicologia: Teoria e Pesquisa, 30, 1-9. doi: $10.1590 / 0102.3772 \mathrm{e} 3321$

Eason, S. H., \& Ramani, G. B. (2017). Parental Guidance and Children's Executive Function: Working Memory and Planning as Moderators During Joint Problem-Solving. Infant and Child Development, 26(2), 1-24. doi: 10.1002/icd.1982

Finegood, E. D., \& Blair, C. (2017). Poverty, parent stress, and emerging executive functions in young children. In K. Deater-Deckard \& R. Panneton (Eds.), Parental Stress and Early Child Development: Adaptive and Maladaptive Outcomes (pp. 181-207). New York: Springer. doi: 10.1007/978-3-319-55376-4_8 
Gueron-Sela, N., Wagner, N. J., Propper, C. B., Mills-Koonce, W. R., Moore, G. A., \& Cox, M. J. (2017). The Interaction Between Child Respiratory Sinus Arrhythmia and Early Sensitive Parenting in the Prediction of Children's Executive Functions. Infancy, 22(2), 171-189. doi: 10.1111/infa.12152

Hackman, D. A., Farah, M. J., \& Meaney, M. J. (2010). Socioeconomic status and the brain: Mechanistic insights from human and animal research. Nature Reviews Neuroscience, 11(9), 651-659. doi: 10.1038/nrn2897

Hammond, S. I., Müller, U., Carpendale, J. I. M., Bibok, M. B., \& Liebermann-Finestone, D. P. (2012). The effects of parental scaffolding on preschoolers' executive function. Developmental Psychology, 48(1), 271-281. doi: 10.1037/a0025519

Hoghughi, M. (2004) Parenting: An introduction. In M. Hoghughi \& N. Long (Eds.), Handbook of parenting: Theory and research for practice (pp. 1-18). London: Sage.

Holochwost, S. J., Gariépy, J. L., Propper, C. B., Neblett, N. G., Volpe, V., Neblett, E., \& Mills-Koonce, W. R. (2016). Sociodemographic risk, parenting, and executive functions in early childhood: The role of ethnicity. Early Childhood Research Quarterly, 36, 537-549. doi: 10.1016/j.ecresq.2016.02.001

Holochwost, S. J., Volpe, V. V., Gueron-Sela, N., Propper, C. B., \& Mills-Koonce, W. R. (2018). Sociodemographic risk, parenting, and inhibitory control in early childhood: The role of respiratory sinus arrhythmia. Journal of Child Psychology and Psychiatry and Allied Disciplines, 59(9), 973-981. doi: 10.1111/jcpp.12889

Hughes, C., \& Devine, R. T. (2017). For Better or for Worse? Positive and Negative Parental Influences on Young Children's Executive Function. Child Development, 90(2), 593609. doi: 10.1111/cdev.12915

Kamza, A., Putko, A., \& Złotogórska, A. (2016). Original Papers Maternal Parenting Attitudes and Preschoolers' Hot and Cool Executive Functions. Polish Psychological Bulletin, 47(2), 236-246. doi: 10.1515/ppb-2016-0028

Kok, R., Lucassen, N., Bakermans-Kranenburg, M. J., van IJzendoorn, M. H., Ghassabian, A., Roza, S. J., ... Tiemeier, H. (2014). Parenting, corpus callosum, and executive function in preschool children. Child Neuropsychology: A Journal on Normal and Abnormal Development in Childhood and Adolescence, 20(5), 583-606. doi: $10.1080 / 09297049.2013 .832741$

Lam, C. B., Chung, K. K. H., \& Li, X. (2018). Parental warmth and hostility and child executive function problems: A longitudinal study of Chinese families. Frontiers in Psychology, 9(1063), 1-12. doi: 10.3389/fpsyg.2018.01063 
Landry, S. H., Miller-Lon car, C. L., Smith, K. E., \& Swank, P. R. (2002). The role of early parenting in children's development of executive processes. Developmental Neuropsychology, 21(1), 15-41. doi: 10.1207/S15326942DN2101_2

Lee, M. K., Baker, S., \& Whitebread, D. (2018). Culture-specific links between maternal executive function, parenting, and preschool children's executive function in South Korea. British Journal of Educational Psychology, 88(2), 216-235. doi: 10.1111/bjep.12221

Lucassen, N., Kok, R., Bakermans-Kranenburg, M. J., Van Ijzendoorn, M. H., Jaddoe, V. W. V., Hofman, A., ... Tiemeier, H. (2015). Executive functions in early childhood: The role of maternal and paternal parenting practices. British Journal of Developmental Psychology, 33(4), 489-505. doi: 10.1111/bjdp.12112

Macarini, S. M., Martins, G. D. F., de Minetto, M. F. J., \& Vieira, M. L. (2010). Práticas parentais: Uma revisão da literatura brasileira. Arquivos Brasileiros de Psicologia, 62(1), 119-134. Recuperado de http://pepsic.bvsalud.org/pdf/arbp/v62n1/v62n1a13.pdf

Martins, G. L. L., León, C. B. R., \& Seabra, A. G. (2016). Estilos parentais e desenvolvimento das funções executivas: Estudo com crianças de 3 a 6 anos. Psico, 47(3), 216-227. doi: 10.15448/1980-8623.2016.3.22480

Merz, E. C., Landry, S. H., Montroy, J. J., \& Williams, J. M. (2017). Bidirectional Associations Between Parental Responsiveness and Executive Function During Early Childhood. Social Development, 26(3), 591-609. doi: 10.1111/sode.12204

Meuwissen, A. S., \& Carlson, S. M. (2015). Fathers matter: The role of father parenting in preschoolers' executive function development. Journal of experimental child psychology, 140(11), 1-15. doi: 10.1016/j.bbamem.2015.02.010.Cationic

Meuwissen, A. S., \& Englund, M. M. (2016). Executive Function in At-Risk Children: Importance of Father-Figure Support and Mother Parenting. Journal of Applied Developmental Psychology, 44, 72-80. doi: 10.1016/j.appdev.2016.04.002

Mileva-Seitz, V. R., Ghassabian, A., Bakermans-Kranenburg, M. J., van den Brink, J. D., Linting, M., Jaddoe, V. W. V., ... van Ijzendoorn, M. H. (2015). Are boys more sensitive to sensitivity? Parenting and executive function in preschoolers. Journal of Experimental Child Psychology, 130, 193-208. doi: 10.1016/j.jecp.2014.08.008

Rolan, E. P., Schmitt, S. A., Purpura, D. J., \& Nichols, D. L. (2018). Sibling presence, executive function, and the role of parenting. Infant and Child Development, 27(e2091), 1-18. doi: 10.1002/icd.2091 
Sarsour, K., Sheridan, M., Jutte, D., Nuru-Jeter, A., Hinshaw, S., \& Boyce, W. T. (2011). Family socioeconomic status and child executive functions: The roles of language, home environment, and single parenthood. Journal of the International Neuropsychological Society, 17(1), 120-132. doi: 10.1017/S1355617710001335

Smith, H. J., Kryski, K. R., Sheikh, H. I., Singh, S. M., \& Hayden, E. P. (2013). The role of parenting and dopamine D4 receptor gene polymorphisms in children's inhibitory control. Developmental Science, 16(4), 515-530. doi: 10.1111/desc.12046

Spruijt, A. M., Dekker, M. C., Ziermans, T. B., \& Swaab, H. (2018). Attentional control and executive functioning in school-aged children: Linking self-regulation and parenting strategies. Journal of Experimental Child Psychology, 166, 340-359. doi: 10.1016/j.jecp.2017.09.004

Sulik, M. J., Blair, C., Mills-Koonce, R., Berry, D., \& Greenberg, M. (2015). Early Parenting and the Development of Externalizing Behavior Problems: Longitudinal Mediation Through Children's Executive Function. Child Development, 86(5), 1588-1603. doi: 10.1111/cdev.12386

Treat, A. E., Sheffield Morris, A., Williamson, A. C., Hays-Grudo, J., \& Laurin, D. (2017). Adverse childhood experiences, parenting, and child executive function. Early Child Development and Care, 189(6), 926-937. doi: 10.1080/03004430.2017.1353978

Vandenbroucke, L., Spilt, J., Verschueren, K., \& Baeyens, D. (2017). Keeping the spirits up: The effect of teachers' and parents' emotional support on children's working memory performance. Frontiers in Psychology, 8(512), 1-12. doi: 10.3389/fpsyg.2017.00512

Vernon-Feagans, L., Willoughby, M., Garrett-Peters, P., \& Family Life Project Key Investigators. (2016). Predictors of behavioral regulation in kindergarten: Household chaos, parenting, and early executive functions. Developmental Psychology, 52(3), 430-441. doi: 10.1037/dev0000087

Yu, J., Cheah, C. S. L., Hart, C. H., \& Yang, C. (2018). Child inhibitory control and maternal acculturation moderate effects of maternal parenting on Chinese American children's adjustment. Developmental Psychology, 54(6), 1111-1123. doi: 10.1037/dev0000517

Zelazo, P. D. (2015). Executive function: Reflection, iterative reprocessing, complexity, and the developing brain. Developmental Review, 38, 55-68. doi: 10.1016/j.dr.2015.07.001 


\section{Endereço para correspondência}

\section{Wayson Maturana de Souza}

Rua Visconde de Ouro Preto, 79/302, Botafogo, Rio de Janeiro - RJ, Brasil. CEP 22250-180

Endereço eletrônico: wmaturanapsi@gmail.com

\section{Luiz Fellipe Dias da Rocha}

Rua São Francisco Xavier, 524, bl. F, $2^{\circ}$ andar, sl. 2145, Maracanã, Rio de Janeiro - RJ, Brasil. CEP 20550-900

Endereço eletrônico: luiz.rocha@uerj.br

\section{Rafael Vera Cruz de Carvalho}

Rua São Francisco Xavier, 524, bl F, $10^{\circ}$ andar, sl 10135, Maracanã, Rio de Janeiro - RJ, Brasil. CEP 20550-900

Endereço eletrônico: rafaelvcc01@yahoo.com.br

\section{Ana Carolina Monnerat Fioravanti}

Avenida Sete de Setembro, 85/402, Icaraí, Niterói - RJ, Brasil. CEP 24230-250

Endereço eletrônico: ana@ fioravantiana.org

Recebido em: 09/09/2019

Reformulado em: 20/05/2020

Aceito em: 01/06/2020

\section{Notas}

* Mestre em Psicologia Social pela Universidade do Estado do Rio de Janeiro, aluno de doutorado no Programa de Pós-Graduação em Psicologia Clínica da Pontifícia Universidade Católica do Rio de Janeiro.

** Mestre em Psicologia Social, aluno de doutorado no Programa de Pós-Graduação em Psicologia Social da Universidade do Estado do Rio de Janeiro.

*** Doutor em Psicologia Social pela Universidade do Estado do Rio de Janeiro, Técnico Administrativo II da Universidade do Estado do Rio de Janeiro.

**** Doutora em Psicologia Clínica, Professora Adjunta de Psicologia na Universidade Federal Fluminense.

Financiamento: Bolsa de Mestrado CAPES ( $1^{\circ}$ Autor)

Este artigo de revista Estudos e Pesquisas em Psicologia é licenciado sob uma Licença Creative Commons Atribuição-Não Comercial 3.0 Não Adaptada. 